\title{
Chemins de fer et déplacements autour de Paris pendant la crise de 1870-1871
}

Bernard Lecoy

\section{OpenEdition}

\section{Journals}

Édition électronique

URL : https://journals.openedition.org/rhcf/698

DOI : $10.4000 /$ rhcf.698

\section{Éditeur}

Rails \& histoire

\section{Édition imprimée}

Date de publication : 1 mai 2004

Pagination : 181-200

ISSN : 0996-9403

\section{Référence électronique}

Bernard Lecoy, «Chemins de fer et déplacements autour de Paris pendant la crise de 1870-1871 »,

Revue d'histoire des chemins de fer [En ligne], 31 | 2004, mis en ligne le 08 avril 2011, consulté le 22 avril 2022. URL : http://journals.openedition.org/rhcf/698 ; DOI : https://doi.org/10.4000/rhcf.698 


\section{Bernard LECOY}

\section{Chemins de fer et déplacements autour de Paris pendant la crise de 1870-1871}

\section{L'investissement}

La capitulation de l'armée de Mac-Mahon à Sedan (2 septembre 1870) ouvrait aux Allemands la route de Paris. Cette situation entraîna une recrudescence de l'activité ferroviaire, particulièrement sur le Nord.

«Pendant ces quatre journées (5-8 septembre) le mouvement sur les lignes du Nord avait pris des proportions colossales ${ }^{1} »$ : militaires ayant échappé à la capitulation, ou dispersés dans les départements du nord-est de la capitale, et repliés sur Paris. « Enfin, à cette même date, le transport des voyageurs devenait plus actif qu'il n'avait jamais été. Aux départs de Paris, l'expulsion des Allemands, l'émigration des bouches inutiles rendaient nécessaire le dédoublement de la plupart des trains, principalement de ceux du soir, qu'on était obligé de faire suivre de deux et quelquefois trois trains spéciaux. L'affluence n'était pas moins grande, d'autre part, aux trains se dirigeant vers Paris. Dans les gares, devenues toutes bien trop petites, les accumulations de bagages formaient de véritables montagnes. Les habitants de la banlieue venaient en masse se réfugier dans la capitale ; des approvisionnements aussi arrivaient en quantité considérable et pourtant insuffisante, hélas ${ }^{2}$ !»

On peut penser que les Parisiens allaient se réfugier en province, tandis que les gens de banlieue s'estimaient plus en sûreté derrière les fortifications de la capitale.

À la gare d'Orléans, l'activité était quelques jours plus tard tout aussi débordante :

«Dans les derniers jours qui précédèrent l'investissement, la Compagnie d'Orléans contribua pour sa part au mouvement énorme des marchandises, denrées, bétail, munitions qui venaient s'engouffrer dans Paris, et au mouvement non moins considérable

1- Baron Ernouf, Histoire des chemins de fer français pendant la guerre franco-prussienne, 1870-1871, Paris, Librairie générale, 1874, 454 p. (texte reproduit en fac-similé, avec illustrations nouvelles, La Vie du rail / Éditions du Layet, 1980).

2- Ibid. 
d'émigration qui se produisit en sens inverse. Il y eut là des scènes de tumulte, d'encombrement, dont ceux qui en ont été les témoins ne perdront jamais le souvenir ${ }^{3}$ !»

L'Illustration racontait la chose avec plus de détachement:

«Plus la note guerrière s'accentue, plus les départs des bouches inutiles se multiplient et prennent des proportions fabuleuses. Qui n'a pas vu les gares de chemin de fer n'a rien vu.

Nous avons tenu à représenter l'aspect de la gare d'Orléans pendant les derniers jours de ces sorties galopantes. Voyez ces montagnes de colis. Elles atteignent le deuxième étage et Bilboquet lui-même, avec toute son habileté à retrouver sa malle, y perdrait son latin.

Pour les billets, c'est mieux encore. On vous les donne comme aux bureaux des omnibus ; mais pour partir Dieu sait quand! On part quand on peut, et, quant aux bagages, on se demande comment les compagnies pourront s'y reconnaitre ${ }^{4}$.»

Ces scènes d'« émigration» (nous dirions d'exode) nous sont confirmées par le rapport du conseil d'administration de l'Orléans (2 août 1871), qui précise même qu'elles se produisaient aussi à la gare de Montrouge (ligne de Limours!) et que certains emportaient avec eux « lits, mobilier et de telles masses de bagages qu'il fallut en suspendre l'expédition, pour maintenir la continuité du service ».

Remarques semblables de la part du conseil d'administration du PLM (3 août 1871).

Cependant, les troupes allemandes approchaient de la capitale. Le 12 septembre, la gare de l'Est avait cessé ses expéditions. Le dernier train, parti de la gare d'Orléans au début de l'après-midi du 16, dut rebrousser chemin à Ablon.

Le 18, quelques trains ont pu s'évader de Paris à travers la fusillade de Clamart, mais le 19 l'encerclement de la capitale était achevé.

«Le chef de la station de Versailles-Chantiers n'avait même pas pris la précaution de démonter son télégraphe, encore en communication avec Paris. Aussi, l'on ne fut pas peu surpris, à la gare Saint-Lazare, en recevant, dans la soirée du 18, un télégramme en style franco-tudesque qui invitait facétieusement la Compagnie de l'Ouest à reprendre le service sur Versailles ${ }^{5}$. »

3- Ibid.

4- Reportage daté du 12 septembre.

5- Baron Ernouf, Histoire des chemins de fer francais..., op. cit. 
Devant l'avance ennemie, le génie français avait fait détruire un grand nombre de ponts ferroviaires et routiers, y compris plusieurs ponts ferroviaires sur le fossé des fortifications à Paris, sites qui n'étaient pourtant pas menacés dans l'immédiat.

À noter que ni le viaduc du Pecq, ni le pont d'Asnières, n'ont à ce moment subi de dommages, bien que la plupart des ponts routiers (ou peut-être tous ?) de ce premier méandre de la Seine aient été détruits.

\section{Paris assiégé}

L'Illustration du 8 octobre 1870 nous décrit gaillardement Paris en guerre : «Les lycées, les édifices publics, les gares de chemin de fer sont transformés en casernes. Les wagons des chemins de fer font d'excellents dortoirs. Chaque quartier de la ville, chaque habitant prend, si l'on peut ainsi dire, son poste de combat. »

Mais le rapport de la Compagnie d'Orléans, déjà cité, nous donne la description la plus complète de la participation du chemin de fer à la défense commune :

«Nos ateliers venant en aide aux arsenaux de l'État, ont réparé des armes, fabriqué des canons, des batteries blindées et des affûts. Deux moulins installés dans nos gares de Paris et d'Ivry ont mis en mouvement trente paires de meules et trituré 27334 quintaux métriques de grains pour la fabrication du pain, pendant les dernières semaines du siège. Nos pompes à vapeur ont servi à alimenter la ville. Vingt-cinq mille moutons ont été parqués, pendant deux mois dans la gare de marchandises d'Ivry. Nos chevaux ont travaillé tous les jours à la distribution des denrées alimentaires dans la ville et aux remparts, au transport des blessés du champ de bataille dans les diverses ambulances... Notre grande gare de Paris, elle-même, où l'on n'entendait plus le bruit des arrivées et des départs, a été transformée en un vaste atelier de fabrication de ballons d'où partaient par une voie nouvelle, les hardis messagers qui mettaient la ville investie en relation avec la France. »

Le conseil d'administration de la Compagnie de l'Est signalait de son côté (4 novembre 1871) :

« Nos ateliers ont été mis à la disposition du gouvernement pour la fabrication des affûts de canon et la réparation des armes. Trente quatre meules à blé, mues par des machines locomotives, ont été installées en vingt jours dans la gare de La Villette. Nous avons, de plus, comme la plupart des autres compagnies, établi une ambulance dans les salles d'attente de la gare. Cette ambulance a reçu cinquante-neuf malades ou blessés. » 
Quant au baron Ernouf, il nous apprend que les ateliers de l'Orléans et de l'Ouest furent particulièrement chargés de la construction des machines et des wagons blindés...

Naturellement, pendant le siège, tous les services de voyageurs avaient cessé de fonctionner, à l'exception des lignes d'Auteuil et de Ceinture (petite ceinture, la seule existant à l'époque) bien qu'on ait entrepris la construction d'une deuxième enceinte le long de la ligne. Leur service ne sera interrompu que les derniers jours des combats de la Commune.

Cependant, l'autorité militaire française a utilisé certains tronçons de ligne, comme celui de Paris à Suresnes : «les bordures en granit des quais des stations sont toutes à replacer; elles avaient été enlevées, sur ordre du général en chef de l'armée de Paris afin que l'on puisse faire circuler des locomotives blindées sur cette partie de la voie ${ }^{6}$. »

C'est sans doute pour la même raison que deux ponts détruits le 17 septembre sur la ligne de Soissons et restés dans la zone française, celui sur le canal Saint-Denis et celui sur le rû de Montfort à La Courneuve, ont été rétablis provisoirement pendant le siège même, en novembre-décembre 1870 .

\section{L'Armistice. État des lieux}

L'armistice du 28 janvier 1871 devait permettre l'élection d'une Assemblée nationale qui déciderait de la reprise des hostilités ou de l'acceptation des conditions prussiennes.

En vertu de l'armistice, les Allemands occupaient les forts de la banlieue qui constituaient la défense de Paris.

Le blocus n'était pas entièrement levé. L'article 9 de la convention précisait que le ravitaillement de Paris devait venir de la zone non occupée.

D'après l'article 10, la sortie des personnes hors de Paris était soumise à des permis réguliers délivrés par l'autorité militaire française et soumis au visa des avant-postes Allemands (et seulement entre six heures du matin et six heures du soir). Et jusqu'au 7 février les habitants des départements voisins de la capitale, venus par route avec des provisions pour leurs parents restés à Paris pendant le siège, étaient repoussés impitoyablement à tous les contrôles.

6- Rapport de l'ingénieur ordinaire du 25 février 1871, archives de la Compagnie de l'Ouest, Archives nationales (Centre des archives du monde du travail, Roubaix), $76 \mathrm{AQ}$. 
En plus des ponts dont la destruction avait été opérée pendant la retraite, plusieurs sections de voies ferrées avaient souffert des opérations ou de leurs conséquences (bien que les dégâts fussent moins étendus que pendant la guerre civile qui allait suivre) :

- la section de Clamart à Meudon, sur la ligne de Versailles-Rive gauche, était endommagée par la construction des batteries prussiennes et par les obus,

- sur celle de Bourg-la-Reine à Sceaux, les Allemands avaient enlevé les rails pour blinder leurs batteries,

- la ligne de Saint-Germain était gravement endommagée entre Nanterre et Rueil,

- enfin, le viaduc du Pecq étant resté seul intact parmi les ponts des environs, routiers ou ferroviaires, les Prussiens avaient transformé la ligne en route sur une longueur d'environ 2500 mètres. "Nous nous trouvons donc en présence d'un travail rendu plus difficile encore par les détritus de matériaux brisés et amalgamés, longtemps foulés par les charrois auxquels cette route était affectée ${ }^{7}$. "

Au moment de l'armistice, les chemins de fer, en zone occupée, étaient pris en mains par l'administration allemande. Celle-ci faisait circuler quelques trains de voyageurs dans des conditions précaires, les têtes de lignes côté Paris se trouvant à Lagny pour l'Est, à Gonesse pour le Nord, en particulier.

Le récit d'un voyageur, parti pour Strasbourg, récit publié dans L'Illustration du 4 mars 1871, nous donne une description de cette situation :

Ce n'est que quatre jours après l'armistice, le 3 février « que j'ai pu, personnellement, et après combien de difficultés et de fins de nonrecevoir, être muni d'un laissez-passer et des informations nécessaires pour entreprendre le voyage... ».

«La première difficulté était d'atteindre les têtes de lignes de chemin de fer, occupées et exploitées par l'Administration prussienne, et les soldats et les employés allemands, au lieu et place de l'Administration française dépossédée. Après délibération, au lieu de faire huit lieues à pied pour atteindre Lagny, je résolus, avec quelques compagnons qui s'étaient joints à moi, de faire un circuit par la ligne de Creil et la courbe de Crespy et Reims pour rattraper la ligne directe à Épernay. Il fallait encore gagner le bourg de Gonesse au-delà de Saint-Denis ${ }^{8}$, c'est-à-dire franchir par terre cinq bonnes lieues, et à pied, vu le déplorable état des

7- Rapport du conseil d'administration de l'Ouest, 30 juin 1871, ibid.

8- Saint-Denis, à l'abri des forts, était tenue par les Français pendant le siège. En imposant l'occupation des forts, l'armistice mettait la ville sous le contrôle des Allemands. 
routes détrempées ou défoncées, et la rareté des bêtes de trait dans une ville qui nourrissait depuis trois mois deux millions d'hommes avec de la viande de cheval...»

"À une demie-lieue ou à une lieue des fortifications, l'on trouvait la ligne serrée sur la route, espacée dans la campagne, des soldats allemands chargés de barrer le passage à tout investi non muni d'un laissez-passer. Au milieu de la route, derrière la ligne, assisté de deux soldats, assis devant une petite table de bois, était le sous-officier qui mettait, le premier, au crayon, une première mention du jour et de l'heure, qui vous permettait d'aller jusqu'au premier commandant prussien, chargé de viser et de sceller du sceau prussien le laissez-passer qui devenait ainsi un sauf-conduit toujours sujet à être révisé, à chaque station importante, le long des lignes occupées par l'envahisseur. Après nous être fait jour, non sans difficulté, nous arrivâmes à Saint-Denis à $9 \mathrm{~h}$ du matin ; nous étions partis de Paris à 6 . Entre la première ligne prussienne et Saint-Denis, les voyageurs munis de permis pour rentrer à Paris étaient souvent plus maltraités que nous, bousculés ou poursuivis qu'ils étaient par des cavaliers allemands, montés sur de grands chevaux et la lance au poing, pour arrêter tous les passants. »

Difficultés de ravitaillement à Saint-Denis, mais les voyageurs voient, après trois mois de pain noir, « un peu de pain blanc ».

Gares dévastées et exploitées par les fonctionnaires prussiens, comme à Gonesse...

«Combien de fois, même après avoir attendu, les voyageurs n'ont-ils pas été mis à la porte et poussés par les soldats avec ces mots : «Alle Leute heraus! - tout le monde dehors »...

«C'est au milieu de ces difficultés et de ces amabilités qu'il a fallu exécuter le voyage; lentement, on le conçoit; la commission d'exploitation allemande ne faisait de convois de voyageurs que de jour, de $5 \mathrm{~h}$ du matin à $9 \mathrm{~h}$ du soir, et obligeait les voyageurs à s'arrêter pour reprendre les billets de vingt en vingt lieues à peu près... Nous avons mis 62 heures à faire le voyage de Strasbourg, avec obligation de coucher deux nuits et de se lever toujours à $4 \mathrm{~h}$ du matin... Ajoutez que ces places qu'on vous octroyait de fort mauvaise grâce, il fallait les payer au prix surélevé de premières classe, et qu'il n'y avait pour les voyageurs français que des wagons allemands de troisième classe, dont les vitres étaient totalement absentes... » 
Plus confortable est le voyage de ce journaliste parti assister aux séances de l'Assemblée nationale, à Bordeaux (les élections avaient eu lieu le 8 février. L'assemblée se réunit le 12) ${ }^{9}$ :

«Avec les chemins de fer dans l'état actuel, les laissez-passer qu'on exige, les stations auxquelles on vous condamne, les lenteurs des locomotives accouplées, les encombrements des railways, Bordeaux est au bout du monde. Où est le temps de ce train rapide qui, du soir au matin, vous transportait de la gare d'Orléans à La Bastide ?... Maintenant, il faut, pour aller de la Bastille aux Quinconces, trente heures au moins, quelques ennuis, beaucoup d'arrêts en chemin et beaucoup de patience.

«Le wagon qui vous prend à la gare vous transporte de Paris à La Motte-Beuvron, à travers les lignes prussiennes. Les soldats de M. de Bismarck montent leur faction dans la banlieue, portant leur fusil Dreyse, suspendu par une courroie de cuir et couché sur leur poitrine, comme un enfant qu'on berce. Ils mettent, à Vitry, sur votre laissez-passer quelques mots d'allemand timbrés à l'encre bleue, qui produisent l'effet désastreux de l'estampille sur les livres ».

«Je reconnais d'ailleurs que ces Germains vous imposent ces petits et gros ennuis avec une politesse stricte et d'une façon point blessante. C'est une chose triste à dire, mais la plupart de nos employés d'administration sont beaucoup plus désagréables, lorsqu'ils vous réclament vos billets, que ces conquérants lorsqu'ils examinent vos papiers. »

«Des gens d'Orléans se précipitent sur nous, aux Aubrais, nous demandant des journaux de Paris, des nouvelles. Un journal de Paris, c'est la manne pour ces pays conquis, occupés par l'ennemi, condamnés aux renseignements prussiens, séparés du monde!»

Le train traverse le Cher et le Limousin, le Périgord, puis le Médoc, Libourne.

«Bordeaux est en fête. Bordeaux, la ville coquette, semble mieux attifée encore et plus provocante. La grisette s'est habillée de soie. »

«Tout se coudoie ici et s'y rencontre. On salue cent personnes en passant, toutes célèbres... des uniformes étincelants, des képis effroyablement galonnés, une cohue de généraux et de colonels...

"Vous avez une mine superbe! Allons, je vois que vous n'avez pas beaucoup souffert!" »

9- L'Illustration, récit daté du 20 février 1871. 
«Et qui dit cela ? Les Parisiens, ceux qu'on n'a plus revus depuis l'investissement, ceux qui ont pris le dernier train en toute hâte, ceux qui ont gémi sur nos privations en sablant le Sauterne et en découpant des poulets de grains. On les retrouve tous ici, accablés des malheurs de la Patrie, et répétant que Paris n’aurait pas fini comme il a fini, s'ils avaient été là !»

\section{Premières réparations}

En ce début de février 1871, il fallait réparer les brèches des voies ferrées, procéder au ravitaillement de la capitale, rétablir un service de voyageurs au moins embryonnaire, enfin obtenir de l'administration allemande la restitution de l'exploitation des réseaux.

Les brèches des fortifications étaient assez vite réparées ; d'autre part, l'armée allemande avait réparé provisoirement certains ponts de grande banlieue.

Mais le 25 février, un certain nombre de lacunes subsistaient:

- le viaduc de Nogent sur la Marne,

- les ponts de Pontoise et d'Auvers-sur-Oise,

- les ponts d'Argenteuil, de Bezons et de Chatou, qui avaient particulièrement souffert,

- le pont (sur la Seine) sur la section du PLM reliant Villeneuve-SaintGeorges et Juvisy.

À quoi il fallait ajouter la mise hors service des sections du Pecq à Saint-Germain, de Clamart à Meudon et de Bourg-la-Reine à Sceaux.

\section{Le ravitaillement}

Le premier convoi de ravitaillement est entré dans la gare d'Orléans dans la nuit du vendredi au samedi 4 février à 3 h du matin, nous dit L'Illustration. Il semblait venir de Bretagne, du moins certains de ses wagons. (Le réseau de l'Orléans s'étendait jusqu'à Landerneau.) Le premier convoi de marée, gagnant les Halles, était escorté de gardes nationaux, et des citoyens-soldats, armés de leurs fusils, se tenaient debout sur les voitures chargées...

Le rapport du conseil d'administration de l'Orléans, déjà cité, précise : «Les préliminaires de paix, bien loin de nous soulager, nous mirent aux prises avec des difficultés croissantes. Nous avions, à la fois, à sauver Paris de la famine par un prompt ravitaillement, et à exploiter des lignes occupées par les Prussiens. Les approvisionnements, préparés dès le mois de novembre par les soins du gouvernement à Bordeaux et dans la Basse-Loire, formaient, au commencement de février, le 
chargement de 7000 wagons, sans compter les demandes énormes du commerce libre ; mais les Prussiens occupaient et exploitaient nos lignes depuis Paris jusqu'à Tours et à la Ferté [Saint-Aubin] ; nous ne pouvions faire notre service qu'avec leur agrément et après eux. Lorsque le ravitaillement de la capitale, par notre ligne, commença le 3 février, les Allemands ne nous permirent que 4 trains dans chaque sens; ce nombre fut porté à 11, à partir du 6 février, et l'exploitation mixte de nos lignes dura ainsi dans des conditions déplorables jusqu'au 13 mars. »

Toujours pour les besoins du ravitaillement, le Nord avait expédié de Paris, dès le $1^{\text {er }}$ février, cinq machines et un grand nombre de wagons.

À la différence de l'Orléans, une grande partie du réseau du Nord se trouvait en zone occupée (seuls le Nord et le Pas-de-Calais avaient échappé à l'invasion) et, au cours de la retraite, on s'était efforcé de soustraire le plus de matériel possible à l'ennemi.

Pendant plusieurs semaines, les Allemands entravèrent la marche des trains de ravitaillement, même expédiés par le département du Nord non occupé : le 4 mars encore, pas moins de 42 trains, comptant 1800 wagons pleins, étaient bloqués à Lille.

En effet, les premières semaines, le seul itinéraire autorisé joignait Dieppe à Paris, par Rouen, Amiens et Creil...

C'est sans doute ce chemin que suivirent les convois de vivres offerts aux Parisiens par les habitants de Londres, et dont nous parle L'Illustration du 11 février. Le premier de ces convois entrait le dimanche 5 en gare du Nord.

Cependant ce fut le réseau de l'Ouest qui fit le plus gros effort dans ce domaine. Comme pour le Nord, les renseignements nous sont fournis par le baron Ernouf.

Dès le début de décembre, des trains de vivres avaient été formés dans la presqu'île du Cotentin. À partir du 6 février, les Allemands autorisaient l'utilisation des lignes de Cherbourg, Granville et Brest. Le premier itinéraire était rabattu sur Argentan et Dreux, et tous trois aboutissaient aux Batignolles, dont la gare ne tarda pas à être encombrée, la rareté des chevaux ne facilitant pas l'enlèvement des denrées.

Au total, jusqu'au 11 février, 82 trains étaient arrivés aux Batignolles : 41, venant de Dieppe, à la gare du Nord (comme je l'ai mentionné plus haut - quelle importance avait dans ce lot le ravitaillement venu d'Angleterre ?) ; enfin sept trains, partis de Brest, étaient acheminés sur les voies de l'Orléans... 
Tous les réseaux ont participé au ravitaillement de Paris, mais les renseignements les plus précis que j'ai pu relever concernent le Nord et l'Ouest.

\section{Reprises précaires}

Comme le ravitaillement, la reprise d'un service régulier de voyageurs dépendait de l'état des lignes, de la bonne volonté des Allemands... et de l'échec aux élections des partisans de la guerre à outrance. Naturellement, les voyageurs devaient être munis d'un laissezpasser pour gagner la zone occupée ou en venir.

Dans la première quinzaine de février, étaient rétablis les services suivants :

- Paris-Orléans, le 7 février,

- Paris-Nevers, le 9 février (service supprimé le 11 et rétabli le 22),

- Paris à Saint-Ouen-l'Aumône, le 8 février.

«Nous croyons pouvoir annoncer qu'à partir d'après demain jeudi, il partira quatre trains par jour de la gare du Nord à destination de :

- Creil, Amiens, Rouen, Dieppe,

- Lille,

- Valenciennes,

- Calais.

Ces trains passeront par la ligne de Pontoise, mais les voyageurs seront obligés de traverser à pied le pont de l'Oise à Auvers. On n'acceptera, dans tous ces trains, même dans ceux de banlieue, que des bagages à mains...

La vérification des laissez-passer se fera à Pontoise ${ }^{10} »$.

D’après un rapport de décembre 1871 , ce service a effectivement débuté ce même samedi 11 février.

En effet, le pont de bateaux provisoire, jugé trop peu solide pour supporter les machines, était utilisé de la manière suivante : une locomotive refoulait le train à partir d'une rive ; une autre le reprenait sur la rive opposée. Cette manœuvre a été effectuée, semble-t-il jusqu'au milieu de mars.

Un service était rétabli sur la ligne de Versailles-Rive droite à partir du 10 février. Les autorités gouvernementales prussiennes résidant alors dans la ville, les services allemands s'étaient réservé la direction de l'exploitation depuis Asnières : départ de Paris à $8 \mathrm{~h}$ du matin et $1 \mathrm{~h}$

10- Journal des chemins de fer, 11 février 1871. 
du soir ; retour à $10 \mathrm{~h}$ du matin et $4 \mathrm{~h} 30$ du soir. La compagnie ne transportait pas les bagages. Les laissez-passer n'étaient délivrés qu'aux catégories suivantes :

1 - ceux qui étaient appelés à Versailles pour une mission d'ordre administratif, politique ou diplomatique,

2 - ceux qui y avaient leur domicile,

3 - ceux dont les père, mère, époux ou enfants y étaient établis.

«À dater du 12 février 1871 :

- un train de transit de Paris [Saint-Lazare] à Rennes, avec retour, ne desservant aucune gare entre Paris et Sillé-le-Guillaume ;

- un train de poste allemand, de Versailles [Chantiers] au Mans avec retour, s'arrêtant à toutes les gares ${ }^{11}$. »

Enfin, à partir du 26 février, un service était rétabli entre Paris et Rouen avec, à Bezons, l'obligation de traverser la Seine en bateau. Le passage des trains sur une voie provisoire était possible à partir du 13 mars. Mais à cette date, on était près d'une nouvelle interruption du trafic...

\section{Les préliminaires de paix}

Le 26 février, après cinq jours de négociations entre Thiers et Bismarck, étaient signés à Versailles les préliminaires de paix : la France cédait l'Alsace-Lorraine. Pour garder Belfort, Thiers avait dû accepter l'entrée des troupes allemandes dans Paris jusqu'à la ratification du traité, ce qui contribua à exaspérer la population parisienne.

Les préliminaires furent ratifiés par l'assemblée de Bordeaux le $1^{\text {er }}$ mars. L'article 3 stipulait : « immédiatement après la ratification les Allemands quitteront l'intérieur de la ville de Paris, les forts situés sur la rive gauche de la Seine et dans le plus bref délai possible tous les territoires... situés sur la rive gauche de la Seine... » L'Assemblée décidait de se transporter à Versailles (10 mars).

Conformément à une convention signée à Ferrières le 9 mars, les Allemands rendaient aux compagnies l'exploitation de leurs lignes : au PLM et à l'Orléans le 13 mars, au Nord vers le 15, enfin à l'Est, qui avait été partagé entre cinq directions allemandes, entre le 17 et le 24.

Au cours de ce même mois, la réparation des voies ou la levée des exigences allemandes avaient permis la reprise du service sur de nouvelles lignes :

- Paris-Montparnasse à Rennes le 6 mars,

- Paris à Limours le 19 mars (sauf l'embranchement de Sceaux).

11- Ibid., 18 février 1871. 
Sur l'Est :

- Paris à Avricourt (nouvelle frontière franco-allemande) le 21 mars,

- Paris à Nogent (le Perreux) le 21 mars.

- Nogent (le Perreux) à Gretz le 4 avril.

Sur le Nord :

- Paris à Creil par Chantilly le 15 mars,

- Paris à Soissons le 21 mars,

- Saint-Ouen-l'Aumône à Pontoise le 4 mai,

- Ermont à Argenteuil le 8 mai, sans liaison vers Saint-Lazare, pour deux raisons « incontournables » : la destruction du pont, les combats de la Commune.

La reprise progressive de la circulation n'excluait pas le retour des accidents ferroviaires. Témoin ce récit de L'Illustration du 18 mars 1871 :

«Un convoi de malades et de blessés allemands, conduit par des employés de la Compagnie de l'Ouest se rendait du Mans à Pantin, pour continuer sur l'Allemagne. Il comportait 32 wagons contenant chacun 20 à 25 hommes.

À 7 heures, un train de banlieue en retard stationnait à Puteaux. Le convoi réussit à s'arrêter, et le chef de gare se mit en devoir de faire les signaux indiquant que la voie n'était pas libre.

Arrive à toute vapeur un train de marchandises (les signaux n'étaient-ils pas faits ?) qui vient heurter les derniers wagons du convoi prussien : 19 wagons broyés avec les malheureux qu'ils contenaient. La locomotive et 5 ou 6 wagons de marchandises également démolis. »

Le journal estimait que le nombre des victimes devait être de 30 à 40 .

\section{La guerre civile}

L'Illustration n'est pas revenue sur l'accident de Puteaux. C'est sans doute que des événements plus graves se produisaient au même moment à Paris. Le 18 mars, à la suite des incidents de Montmartre, l'insurrection se propageait dans Paris. Le gouvernement se retirait à Versailles, abandonnant aux insurgés la capitale, et même les forts de la rive gauche, sauf le Mont-Valérien (ceux de la rive droite étaient occupés par les Allemands).

La guerre civile provoqua un nouvel exode de la population parisienne. « La première semaine de la Commune a produit le même effet que l'arrivée de l'armée prussienne, une émigration énorme. Certains quartiers sont devenus presque déserts. On n’évalue pas à moins 
de 150000 le nombre des personnes qui ont pris le chemin de fer. La fermeture des portes a brusquement arrêté le sauve-qui-peut de la peur », nous dit L'Illustration du 8 avril 1871.

Cependant les relations ferroviaires des deux lignes entre Paris et Versailles avaient été suspendues; la ligne du Havre avait son origine reportée à Maisons-Laffitte (celles de Saint-Germain et d'Argenteuil n'avaient pas semble-t-il, été rouvertes, même partiellement). Quant aux lignes de l'Orléans, le trafic était interrompu le 4 avril entre Paris et Limours, le 12 entre Paris et Juvisy.

Seules continuaient à fonctionner les lignes se dirigeant vers la zone occupée par les Allemands, avec des restrictions imposées par les autorités de la Commune.

La politique de celles-ci à l'égard des fuyards semble avoir été incertaine : L'Illustration du 22 avril note encore : « chaque jour la population fuit, chaque jour, elle s'en va, si bien qu'on se demandera si Paris est habité. » Le même numéro ajoute : «à partir du 15 avril, $8 \mathrm{~h}$ du matin, le service des passeports est organisé dans la mairie de chaque arrondissement. Toutes personnes qui désirent des laissez-passer ou passeports, et qui ne se trouvent pas sous le coup de la loi militaire communale, pourront donc les obtenir à leur mairie. Les maires mettront à cet effet un local à la disposition des fondés de pouvoirs du délégué civil de l'ex-préfecture. »

Naturellement, ceux qui se trouvaient sous le coup de cette loi militaire n'étaient pas forcément désireux d'être incorporés dans l'armée de la Commune, et le même journal nous décrit quelques moyens employés par des jeunes qui, à la gare du Nord, cherchaient à échapper aux « cerbères » qui gardaient la porte de la salle d'attente; jeunes que le journal surnomme les «francs-fileurs »: l'un se déguise en Anglais, « jumelles en sautoir, chapeau américain, châles sur épaules, passeport en règle, tenue britannique irréprochable »; l'autre se cache dans une armoire de déménagement; un troisième s'habille en jeune fille, et « justifie ses doigts jaunis par l'habitude du tabac partagée par toute sa famille. »

Si les autorités de la Commune ne mettaient que peu de restrictions à la circulation des trains de voyageurs, sinon à celle des voyageurs euxmêmes, au départ des trois réseaux déjà cités (Nord, Est, PLM), il n'en était pas de même du service des marchandises. À la fin d'avril, en effet, elles interdisaient tout départ de Paris de wagons de marchandises ou de machines haut-le-pied ${ }^{12}$. La composition des trains de voyageurs

12- Ibid., 29 avril 1871. 
devait être réduite au nombre de véhicules nécessaires pour le nombre de voyageurs en partance. Les autorités s'apercevaient, peut-être un peu tard, que tout matériel quittant Paris pouvait contribuer à accélérer le rapatriement des prisonniers, sur lesquels le gouvernement comptait pour renforcer son armée.

D'ailleurs, le 4 mai, cette interdiction était assouplie : elle ne concernait plus que les vivres, farines, liquides ou denrées alimentaires, les équipements militaires, armes et munitions de guerre ${ }^{13}$.

Pendant ces événements, les compagnies, en particulier celle du Nord, se sont efforcées de tenir leur personnel à l'écart de la contagion révolutionnaire. « Nous avons formé, pour la défense de nos établissements un bataillon de garde nationale composé des agents et des ouvriers des gares de Paris et de La Chapelle. Outre les avantages de sécurité que cette mesure nous a procurés, nous avons pu aussi maintenir parmi notre nombreux personnel des habitudes d'ordre, d'assiduité et de discipline ${ }^{14} . »$

Le 27 mai 1871, c'est à dire un des derniers jours de la guerre civile, L'Illustration publiait un récit intitulé : « Les environs de Paris pendant la guerre civile » dont voici de longs extraits :

«... à plus de trente lieues à la ronde, si vous vous présentez, en quête d'un logis, dans un hôtel ou dans une auberge, on vous répondra invariablement que votre recherche est vaine, que tout est pris par les Parisiens. »

«Versailles est, naturellement, le quartier général de l'émigration; c'est le Coblentz de ce nouveau 93 et il y aurait tout un volume à faire sous ce titre. Un voyage de Paris à Versailles, sous le règne de la Commune, en l'an de grâce 1871 car ce voyage coûte plus de temps et de fatigue qu'il n'en faut d'ordinaire pour aller au Havre ou à Bruxelles... »

«Les deux lignes de l'Ouest étant coupées, il faut, pour sortir de Paris, prendre le chemin de fer du Nord et se rendre à SaintDenis, d'où l'on peut gagner Versailles en passant par Nanterre et Bougival. Je ne mentionnerai que pour mémoire les difficultés qu'il faut surmonter pour franchir la porte de la salle d'attente, que gardent les cerbères de la Commune: L'Illustration a déjà parlé des moyens plus ou moins ingénieux employés par les réfractaires qui cherchent à se soustraire à la levée en masse. Nous

13- Le ravitaillement de la ville se faisait désormais à partir de la zone occupée. Il ne fallait évidemment pas retenir tout le matériel qui pouvait y contribuer.

14- Rapport du conseil d'administration du Nord, 30 juin 1871. 
sommes à Saint-Denis. Au sortir de la gare, on est assailli par une nuée d'individus qui se disputent votre personne, et qui vous assourdissent de leurs cris pour vous proposer une place dans quelque tapissière en partance.»

«Les voitures ne manquent pas, du reste; il y en a de toutes formes, de toutes dimensions, et à tous les prix, depuis le coupé de remise, jusqu'à la voiture de maraîcher meublée de chaises. Toutes ces voitures s'engagent en une longue file dans la rue qui aboutit à la Seine, dont elle franchit les deux bras sur un double pont suspendu. Des factionnaires prussiens règlent la marche du cortège et veillent à ce que chacun ne passe qu'à son tour, précaution indispensable pour éviter l'encombrement ${ }^{15}$. »

«Le pont de Saint-Denis est un de ceux qui ont eu le rare privilège d'échapper à la destruction au moment de la marche de l'armée allemande sur Paris. Sur le cours seul de la Seine, on ne compte pas moins de 28 ponts brûlés ou démolis par la mine entre Paris et Rouen. Les deux ponts de Chatou ont subi le sort commun. Celui du chemin de fer de Saint-Germain laisse tristement pendre dans les eaux de la rivière les membres de sa charpente brisée ${ }^{16}$. Celui de la route, dont il ne reste que les piles, a été remplacé par un bac, appareil primitif qui fait l'étonnement des passants qui le voient pour la première fois. »

« La Seine, à Chatou comme à Saint-Denis, est la frontière qui limite le territoire occupé par les troupes allemandes; celles-ci ont réoccupé en forces les localités qu'elles avaient d'abord évacuées ${ }^{17}$; tous ces villages ont eu ainsi à subir une seconde fois l'invasion dont ils se croyaient délivrés, et je vous laisse à penser si les habitants ont su gré à la Commune de leur valoir ce nouveau fardeau... »

\section{Opérations et voies ferrées}

Au mois d'avril, les combats firent d'importants dégâts dans la région d'Asnières. Jusqu'au milieu du mois, les Fédérés (ou Communards) tenaient une tête de pont sur la rive gauche du fleuve. Ils lancèrent même sur la voie ferrée des wagons blindés pour aller bombarder Courbevoie. Les 17 et 18 avril, après s'être emparé du château de Bécon, les Versaillais reprennent la ville d'Asnières, au cours de durs combats. Les Fédérés se replient sur Clichy au moyen du pont de bateaux établi pendant le premier siège.

15- La situation a changé depuis le reportage paru le 4 mars.

16- Les arches avaient été reconstruites en tôle en 1864-1865.

17- Repliés au nord de la Seine, les Allemands n'avaient pas estimé utile d'en occuper tous les méandres. 
À leur tour, les Versaillais établissent une batterie d'artillerie au château de Bécon, pour atteindre le pont de chemin de fer et les wagons blindés qui s'y étaient repliés. Les Fédérés, retranchés dans Clichy et Saint-Ouen, dirigent maintenant sur Asnières leurs bombardements meurtriers.

Cependant les Versaillais ont installé une centaine de batteries à quelque distance des limites ouest et sud de Paris, depuis Gennevilliers jusqu'à Choisy-le-Roi : plusieurs étaient établies sur la voie ferrée, à Asnières, Puteaux et Suresnes; au sud, la plupart avait récupéré les emplacements aménagés par les Allemands pour leur propre artillerie.

\section{Bilan des destructions}

Le 21 mai, les Versaillais pénétraient dans Paris par la porte du "Point du jour », au sud-ouest. La Commune était vaincue, après une semaine de combats de rue. Les bombardements, les combats et les incendies allumés par les vaincus ont occasionné d'importantes destructions et le chemin de fer a payé un assez lourd tribut. L'Illustration et les conseils d'administration des compagnies nous en donnent d'assez longues descriptions.

«À la porte Maillot... Ce n'est pas pour rien qu'ont travaillé de ce côté, deux mois durant, les canons du Mont-Valérien, du château de Bécon, du Rond-point de Courbevoie ${ }^{18}$... et d'abord, inutile de chercher la gare; vous n'en verrez même plus les restes, ils ont été enlevés. Ce large et profond fossé, à moitié rempli de débris de toutes sortes, c'est le tunnel. Voilà ce que la guerre civile en a fait...

«Le viaduc du Point-du-jour, a été moins éprouvé qu’on aurait pu le croire, exposé qu'il était au feu incessant des puissantes batteries du sud. La gare, chose curieuse dans la position dominante qu'elle occupe, en a été quitte pour la perte de son vitrage.

«En montant vers Auteuil, le mal devient plus grave. Les maisons bordant le boulevard Exelmans ont été gravement atteintes... À la gare d'Auteuil, c'est lamentable.

«Le tablier de fer qui joint la gare au viaduc est brisé. De la gare elle-même, il ne reste plus que des débris. Le zinc de son toit, cent fois touché et fléchissant, laisse pendre ses grandes feuilles comme les ailes d'un oiseau blessé. Ruine qu'entoure un cercle de ruines ${ }^{19} \ldots »$

18- Depuis lors rond-point de La Défense.

19- L'Illustration, 17 juin 1871. 
À Asnières, le joyeux centre du canotage parisien, tout est en ruine. La gare n'existe plus. Quant au pont :

« la charpente métallique seule est en place, inébranlable, portant toujours les rails sur son dos robuste. Mais elle est à jour. Ce n'est plus qu'un long grillage, entre les barreaux duquel, quand un train traverse la Seine, on peut voir au-dessus de soi se refléter dans l'eau, entraîné par la locomotive, le lourd chapelet des wagons ${ }^{20}$.»

Le rapport du conseil d'administration de l'Ouest du 30 juin 1871 précise :

«Enfin le pont d'Asnières, si souvent menacé pendant la guerre et que nous avions sauvé de la destruction, a été miné par les insurgés, qui ont tenté de le faire sauter au moyen de poudre introduite dans les tubes qui forment les poutres. Cet acte de sauvagerie n'a heureusement pas réussi complètement ; néanmoins la travée la plus rapprochée de Paris a été gravement détériorée et nous avons dû la consolider provisoirement de manière à permettre le passage des trains, en attendant une réparation définitive. »

Le même rapport signale des destructions plus ou moins importantes à la gare des Batignolles, à celles de Clichy-Levallois, Grenelle (sur la Ceinture), Ouest-ceinture et Clamart, aux passages supérieurs situés entre ces deux gares, enfin aux ateliers de Bois-Colombes.

Il faut ajouter que les gares d'Orléans, de l'Est, Montparnasse, et surtout celle de Lyon, avaient été en partie incendiées, en partie saccagées et trouées par les obus (comme d'autres bâtiments publics ; on sait qu'à la place de la Cour des comptes incendiée, on édifiera la gare d'Orsay).

Quant à la gare d'Argenteuil, et à celle des fêtes de Saint-Cloud, elles avaient déjà été incendiées par les Allemands... La seconde, d'ailleurs, ne sera jamais reconstruite.

\section{Le retour de la paix}

La circulation sur la banlieue ouest avait été totalement interrompue par la guerre civile. Utilisant parfois des installations provisoires, les différentes lignes furent rendues au service des voyageurs aux dates suivantes :

- Paris à Versailles-Rive droite, le 31 mai. Les trains partaient de deux heures en deux heures (la moitié du service du temps de paix). Les voyageurs devaient être munis d'un laissez-passer (exigé cette fois-ci par le gouvernement français),

- Paris à Rueil, le 2 juin (sur une voie depuis Nanterre jusqu'au 2 septembre),

20- Ibid. 
- Paris à Argenteuil - gare provisoire sur la rive gauche de la Seine) le 2 juin,

- Paris à Versailles-Rive gauche, le 3 juin. Les trains circulaient déjà quelques jours auparavant et servaient au transport des troupes, - Rueil à Saint-Germain le 29 août. La réparation provisoire des ponts de Chatou avait été entreprise pendant la guerre civile et le viaduc du Pecq était déblayé.

Ligne d'Auteuil et de Ceinture :

- de Courcelles à Orléans-Ceinture (par la rive droite) le 30 mai, - d'Ouest-ceinture à Orléans-Ceinture, le 17 juin,

- de Courcelles à Ouest-Ceinture, section la plus endommagée, le 2 juillet.

Sur l'Orléans, le service des voyageurs au départ de Paris était repris le 3 juin. Sur les autres réseaux, comme je l'ai dit, la guerre civile ne l'avait pratiquement pas interrompu. Assez vite, sa consistance retrouvait ses normes d'avant la guerre.

D'ailleurs, comme le signalait l'Orléans, les trains ramenaient chez eux des nombreux Parisiens qui s'étaient réfugiés en province.

À la fin de l'année, le réseau ne présentait plus que deux lacunes : - le pont d'Argenteuil. Les voyageurs venus de Pontoise (comme ceux partant d'Argenteuil) devaient emprunter le pont de la route pour gagner la gare provisoire établie sur la rive gauche. Le pont ne fut livré à la circulation des trains que le 31 mars 1872 ;

- la section de Villeneuve-Saint-Georges à Juvisy sur le PLM. La desserte de Corbeil se faisait au départ de la gare d'Orléans, comme aux premiers temps. Réouverture le 5 février 1872.

Si, dès la fin de l'année 1871, les trois ponts sur l'Oise de la Compagnie du Nord étaient définitivement reconstruits, il subsistait en 1872 de nombreux ouvrages provisoires. Les culées et piles de pont de Bezons étaient reconstruites au début de l'année. Mais pour entreprendre la partie métallique, il a fallu attendre l'achèvement de celui d'Argenteuil, qui permettait d'acheminer les matériaux depuis l'usine Jolly. Le nouvel ouvrage a été ouvert au service des trains le 23 juillet 1872. C'est vers la fin de cette année qu'était terminé également le pont d'Asnières.

Mais la reconstruction définitive des ponts de Chatou était ajournée à plusieurs années ${ }^{21}$. Il en était de même de celle des bâtiments des gares : en particulier de la Porte Maillot, Auteuil, Asnières, Argenteuil et Clamart. «Au nombre des travaux urgents auxquels il resterait encore à pourvoir, nous signalerons le rétablissement des gares de voyageurs

21- Le service se faisait dans de très bonnes conditions sur les ponts provisoires. 
dont le service n'est assuré que par des aménagements provisoires et qui sont l'objet de réclamations incessantes, tant de la part de l'administration que de celle du public ${ }^{22}$. » Il est vrai que le contentieux des indemnités était long à régler...

Cependant, le trafic de banlieue avait repris son ascension, comme le montrent les chiffres de la banlieue ouest ${ }^{23}$ :

- 1869 : 18924000 voyageurs

- 1871 : 12836000 voyageurs

- 1872 : 19823000 voyageurs.

\section{Sources et bibliographie}

L'Illustration, journal universel (vol. 1, n 1 (4 mars 1843) - vol. 215, n 5292/ 5293 (19 août 1944), Paris, Dubochet, années 1870 et 1871.

Le Journal des chemins de fer (paru de 1842 à 1940), années 1870 et 1871.

Baron Ernouf [Alfred-Auguste Ernouf (1817-1889)], Histoire des chemins de fer français pendant la guerre franco-prussienne, 1870-1871, Paris, Librairie générale, 1874, in-18, 454 p.

Collections des rapports du conseil d'administration à l'assemblée générale des compagnies de chemin de fer, Archives nationales (Centre des archives du monde du travail, Roubaix) :

- Compagnie des chemins de fer de l'Ouest, $76 \mathrm{AQ}$

- Compagnie des chemins de fer de l'Est, 13 AQ

- Compagnie des chemins de fer de Lyon à la Méditerranée, 77 AQ et 196 AQ

- Compagnie du chemin de fer du Nord, 48 AQ et 202 AQ.

22- Rapport du conseil d'administration de l'Ouest du 29 mars 1873, AN (CAMT), $76 \mathrm{AQ}$.

23- Ibid. 